\title{
Association of methylenetetrahytrofolate reductase (MTHFR) C677T and A1298C polymorphisms with the susceptibility of childhood acute lymphoblastic leukaemia (ALL) in Chinese population
}

Xiaolei Li', Qingchuan Liao², Shunguo Zhang ${ }^{1}$ and Minling Chen ${ }^{1 *}$

\begin{abstract}
Background: The aim of this study was to investigate the relationship between the polymorphisms of the methylenetetrahytrofolate reductase (MTHFR) gene and susceptibility to childhood acute lymphoblastic leukemia (ALL).

Methods: A case-control study was conducted among 98 children with ALL and 93 age- and sex- matched non-ALL controls. Genotyping of MTHFR C677T and A1298C polymorphisms was performed by polymerase chain reaction-restriction fragment length polymorphism (PCR-RFLP). The odds ratios (ORs) of MTHFR genotypes were used to assess the associations of these polymorphisms with childhood ALL susceptibility.

Results: No significant differences were observed for frequencies of the $677 C C, 677 C T$ and $6771 T$ genotypes between patients and controls. Frequencies of the 1298AA, 1298 AC and 1298CC genotypes between the two groups were significantly different. The risk of ALL with the $1298 \mathrm{C}$ allele carriers $(A C+C C)$ was elevated by 1.1 times compared with the AA genotype $[\mathrm{OR}=2.100 ; 95 \% \mathrm{Cl}(1.149 ; 3.837) ; P=0.015]$.
\end{abstract}

Conclusions: The MTHFR A1298C polymorphism is associated with susceptibility to childhood ALL in the Chinese population.

Keywords: Methylenetetrahydrofolate reductase (MTHFR), C677T, A1298C, Gene polymorphism, Acute lymphoblastic leukemia

\section{Background}

Acute lymphoblastic leukemia (ALL) is a malignant disorder of lymphoid progenitor cells with a peak prevalence at the ages 2-5 years old [1] and accounts for one third of all childhood malignancies [2,3]. Although the rapid progress in the clinical diagnosis and effective treatment of childhood ALL has led to a cure rate of more than $80 \%$ in children [4], the precise pathogenesis of ALL is not clearly known. With the development of genetic pharmacology, people have gradually realized that individual differences in disease are mainly due to genetic polymorphisms, especially single nucleotide polymorphisms (SNPs) [5]. SNPs, a

\footnotetext{
* Correspondence: minling-chen@163.com

'Department of Pharmacy, Shanghai Children's Medical Center, Shanghai Jiaotong University School of Medicine, 1678 Dongfang Road, Pudong, Shanghai 200127, China

Full list of author information is available at the end of the article
}

type of polymorphism involving variation of a single base pair, may directly affect the structure or expression of the protein. Therefore, the characterization and detection of the SNP distribution differences in different populations will help to identify human susceptibility genes related to some diseases.

Polymorphisms of the methylenetetrahydrofolate reductase (MTHFR) gene were shown to be associated with leukemogenesis [6]. MTHFR is a folate metabolic enzyme, catalyzing the reduction of 5,10-methylenetetrahydrofolate to 5-methyltetrahydrofolate [7,8]. Two common SNPs of the MTHFR gene, C677T and A1298C, have been identified. MTHFR C677T converts alanine into valine at codon 222, and A1298C converts glutamic acid into alanine at codon 429. Changes in these two loci can lead to decrease of enzyme activity and plasma folate level, as well as homocysteine level elevation [9]. Associations of the 
polymorphisms with a number of cancers, including prostate cancer, colorectal cancer, renal carcinoma and bladder cancer, have also been proposed $[8,10]$. Recently, the relativity between MTHFR polymorphisms and susceptibility to childhood ALL has come under intense scrutiny [2,6,11-16]. Because the MTHFR gene distribution varies among different populations $[3,6]$, although relationships of MTHFR polymorphisms and leukemia in different populations have been reported [2,6,11-19], whether C677T and A1298C gene polymorphisms are involved in the susceptibility to childhood leukemia remains largely controversial.

In the present study, we used the polymerase chain reaction-restriction fragment length polymorphism (PCRRFLP) technique to investigate whether MTHFR polymorphisms and their allele frequency may be correlated to susceptibility to childhood ALL among the Chinese population.

\section{Methods \\ Patients}

From October 2007 to April 2011, a total of 98 children (54 boys, 44 girls) with ALL were diagnosed by bone marrow cytology in Nanjing Children's Hospital, Nanjing, China. Patient ages ranged from 0.9 to 11 years old (mean, 5.1 years old). Meanwhile, a total of 93 controls (1.213 years old; mean, 5.7 years old) hospitalized during the same period were recruited from clinical departments other than hematology or oncology of Nanjing Children's Hospital. The controls had no blood disease or family history of ALL assessed by medical history and clinical examination. There was no significant difference between the two groups in gender and age distribution $(P>0.05)$. All individuals and/or their parents provided informed consent to participate in this study, and the protocol was approved by the medical research ethics committee of Nanjing Children's Hospital.

\section{Genotyping of MTHFR variants}

MTHFR C677T and A1298C polymorphisms were detected by PCR-RFLP assay as described by Atashrazm et al. [3] with slight modification. A total of $2 \mathrm{ml}$ venous blood was extracted from each subject, and EDTA was used for anticoagulation. The conventional phenolchloroform extraction method was used to extract the genomic DNA. MTHFR gene exon 4 (containing C677T polymorphism site) and exon 7 (including A1298C polymorphic site) were amplified by PCR. Primer sequences and the annealing temperature of PCR are presented in Table 1. For the details, the PCR thermal cycling condition included 35 cycles of $95^{\circ} \mathrm{C}$ for $5 \mathrm{~min}, 94^{\circ} \mathrm{C}$ for $30 \mathrm{~s}$, $56^{\circ} \mathrm{C}$ or $55^{\circ} \mathrm{C}$ (for polymorphic sites at positions 677 and 1298 , respectively) for $1 \mathrm{~min}, 72^{\circ} \mathrm{C}$ for $30 \mathrm{~s}$ and $72^{\circ} \mathrm{C}$ for 8 min. Primers were designed using Premier 5 software and synthesized by the Beijing Genomics Institute (Beijing, China). The primer's specificity was confirmed by BLAST in Genbank.

\section{Restriction endonuclease mapping analysis}

MTHFR C677T: the PCR products were digested by HinfI (TaKaRa, Dalian, China) after overnight incubation at $37^{\circ} \mathrm{C}$. The final products were subjected to electrophoresis on $2 \%$ agarose, and the genotypes were determined by examining the stained gel in $0.2 \%$ ethidium bromide. A fragment of 387 bp indicated a CC wild genotype; 3 fragments of 387, 235 and 152 bp were indicators of a CT heterozygote; a TT homozygous mutant produced two fragments of $235 \mathrm{bp}$ and $152 \mathrm{bp}$, respectively.

MTHFR A1298C: PCR products were digested with MbolI (TaKaRa, Dalian, China). The final products were subjected to electrophoresis on $2 \%$ agarose, and the genotypes were determined by examining the stained gel in $0.2 \%$ ethidium bromide. The AA wild genotype produced five fragments of 56, 31, 30, 28 and $18 \mathrm{bp}$, respectively; the $\mathrm{AC}$ heterozygote produced six of fragments $84,56,31,30,28$ and $18 \mathrm{bp}$, respectively; the CC homozygous mutant produced four fragments of 84, 31, 30 and 18 bp, respectively. GENESCAN ${ }^{\mathrm{m}} 672$ software (Applied Biosystems, Foster City, CA, USA) was used for collecting information from every band, and GeneMapper software (Applied Biosystems, Foster City, CA, USA) was used to analyze the data.

\section{Statistical analysis}

Data were input into Epidata3.0 to establish a database. Statistical analysis was performed by SPSS16.0 (SPSS Inc., Chicago, IL, USA). Hardy-Weinberg equilibrium was assessed by the $x^{2}$ goodness of fit test. Genotype and allele frequencies were expressed by percentile, and the haplotype and linkage disequilibrium were estimated by SHEsis software (Bio-X Life Science Research Center of Shanghai Jiao Tong University, Shanghai, China). The $\mathrm{X}^{2}$ test was used to examine differences in MTHFR genotypes and allele frequencies between cases and controls. The odds ratio (OR) and 95\% confidence interval (CI) were used for correlation analysis of ALL morbidity, and the MTHFR genotype. $P<0.05$ was considered statistically significant.

\section{Results}

\section{Hardy-Weinberg equilibrium test}

Genotype distributions for both markers among control and ALL groups were found to be in HWE (Table 2).

MTHFR C677T genotype and allele frequency distribution Genotype and allele frequency for MTHFR C677T is shown in Table 3. Frequencies of 677CC, 677CT and 677TT were $28.6 \%, 44.9 \%$ and $26 \%$ in the ALL group 
Table 1 Characteristics of PCR primers sequence, target fragment length and annealing temperature used for genotyping MTHFR polymorphisms

\begin{tabular}{lllll}
\hline Polymorphisms sites & & Primers sequence $\left(\mathbf{5}^{\prime} \rightarrow \mathbf{3}^{\prime}\right)$ & Fragment length & Annealing temperature \\
\hline MTHFR C677T & F & AGTCCCTGTGGTCTCTTCATC & $387 \mathrm{bp}$ & $56^{\circ} \mathrm{C}$ \\
MTHFR A1298C & R & GGAGATCTGGGAAGAACTCAG & \\
& F & CTTGGGGAGCTGAAGGACTACTAC & $168 \mathrm{bp}$ & $55^{\circ} \mathrm{C}$ \\
\hline
\end{tabular}

and $31.2 \%, 52.7 \%$ and $16.1 \%$ in the control group, respectively. No significant differences in genotype distributions of MTHFR C677T were found between the ALL group and control group $\left(\chi^{2}=3.109, P=0.211\right)$. The $677 \mathrm{~T}$ allele frequency distribution between the two groups also showed no significant difference $(49.0 \%$ vs. $42.5 \%$, $X^{2}=1.627, P=0.202$ ).

MTHFR A1298C genotype and allele frequency distribution Genotype and allele frequency for MTHFR A1298C is shown in Table 4. Frequencies of 1298CC, 1298CT and $1298 \mathrm{TT}$ were $55.1 \%, 42.9 \%$ and $2.0 \%$ in the ALL group and $72.0 \%, 26.9 \%$ and $1.1 \%$ in the control group, respectively. The overall distributions of MTHFR A1298C genotype frequencies in the two groups were significantly different $\left(X^{2}=5.917, P=0.05\right)$. MTHFR $1298 \mathrm{C}$ allele frequency in the ALL group was significantly higher than that in the control group $\left(23.5 \%\right.$ vs. $14.5 \%, X^{2}=4.949$, $P=0.026)$. Besides, from Table 4 , we find that the OR of genotype CC is too large (0.219-28.104), which might result from the very low number of $\mathrm{CC}$ cases (two cases). Therefore, we combined the genotypes of $\mathrm{AC}$ and $\mathrm{CC}$ to calculate the distribution. The $\mathrm{C}$ allele $(\mathrm{AC}+\mathrm{CC}$ genotype) frequency in the ALL group was significantly higher than that in the control group $\left(44.96 \%\right.$ vs. $28.0 \%, X^{2}=5.898$, $P=0.015)$, and the $C$ allele $(\mathrm{AC}+\mathrm{CC})$ carriers had a 1.1 times higher risk of ALL than the AA genotype carriers $(\mathrm{OR}=2.100,95 \% \mathrm{CI}: 1.149$ - 3.837).

\section{Discussion}

In recent years, many articles have reported on the MTHFR polymorphism and susceptibility to ALL, but they are soundly inconclusive. In this study, by investigating the distribution of MTHFR C677T and A1298C genotypes and their allele frequencies in ALL children and non-ALL controls, we found that MTHFR A1298C was significantly related to the susceptibility to childhood ALL.

In 1999, Skibola et al. [20] first reported that the MTHFR C677T mutation could reduce the susceptibility to adult ALL, and their results suggested that the risk of ALL was reduced by 4.3 times for people carrying the TT genotype. Subsequently, Franco et al. [21] had similar findings that the C677T mutation can reduce the risk of childhood ALL. Later, multiple documents reported a protective effect of the TT genotype on the pathogenesis of ALL [3,6,11,12,]. However, the association is mostly not statistically significant $[3,13]$, and some studies even suggested that the C677T mutation is an ALL risk factor [22]. A meta-analysis including 13 studies with a total of 4,894 individuals indicated that the C677T mutation was not associated with childhood ALL [23]. In this study, we also report a statistically non-significant difference of genotype frequencies among cases and controls, indicating that the MTHFR C677T gene polymorphism has no relation to the risk of childhood ALL. This result is in accordance with the results of Atashrazm et al. [3] and Oliveira et al. [13].

The MTHFR A1298C polymorphism was associated with an elevated risk of childhood ALL [6]. Alcasabas et al. [7] found that the ALL risk of MTHFR 1298C carriers $(\mathrm{AC}+\mathrm{CC}$ type) was 1.57 times higher than that of the AA genotype carriers. Kim et al. [24,25] had similar findings. Other studies in Germany [17], the UK [2], north Indian [18] and Kurdish [19] showed no associations between the MTHFR A1298C polymorphism and risk of ALL. In our study, the risk of ALL in MTHFR 1298 AC genotype carriers was 2.084-fold that of the

Table 2 Compositions of the MTHFR C677T genotype and MTHFR A1298C genotype in the Hardy-Weinberg equilibrium test

\begin{tabular}{lccccc}
\hline & \multicolumn{2}{c}{ MTHFR C677T genotype } & & \multicolumn{2}{c}{ MTHFR A1298C genotype } \\
\cline { 2 - 4 } & ALL group & Control group & & ALL group & Control group \\
\hline CC type & $28(28.6 \%)$ & $29(31.2 \%)$ & AA type & $54(55.1 \%)$ & $67(72.0 \%)$ \\
CT type & $44(44.9 \%)$ & $49(52.7 \%)$ & AC type & $42(42.9 \%)$ & $25(26.9 \%)$ \\
$T$ type & $26(26.5 \%)$ & $15(16.1 \%)$ & CC type & $2(2.0 \%)$ & $1(1.1 \%)$ \\
$X^{2}$ & 1.013 & 0.569 & $X^{2}$ & 3.652 & 0.643 \\
$P$-value & 0.314 & 0.451 & $P$-value & 0.06 & 0.423 \\
\hline
\end{tabular}


Table 3 Frequencies of MTHFR C677T polymorphisms in childhood ALL patients and controls

\begin{tabular}{|c|c|c|c|c|c|}
\hline MTHFR C677T & Patient no. (\%) & Control no. (\%) & $x^{2}$ & $P$ & OR $(95 \% \mathrm{Cl})$ \\
\hline \multicolumn{6}{|l|}{ Genotype } \\
\hline CC & $28(28.6)$ & $29(31.2)$ & & - & $1.00^{\#}$ \\
\hline $\mathrm{CT}$ & $44(44.9)$ & $49(52.7)$ & 0.046 & 0.829 & $0.930(0.481-1.799)$ \\
\hline$\Pi$ & $26(26.5)$ & $15(16.1)$ & 1.969 & 0.161 & $1.795(0.790-4.079)$ \\
\hline$C T+\pi$ & $70(71.4)$ & $64(68.8)$ & 0.155 & 0.693 & $1.133(0.609-2.106)$ \\
\hline \multicolumn{6}{|l|}{ Allele } \\
\hline C & $100(51.0)$ & $107(57.5)$ & & - & $1.00^{\#}$ \\
\hline $\mathrm{T}$ & $96(49.0)$ & $79(42.5)$ & 1.627 & 0.202 & $1.300(0.868-1.947)$ \\
\hline
\end{tabular}

1298AA genotype carriers $(\mathrm{OR}=2.084,95 \% \mathrm{CI}: 1.131-$ 3.841, $P=0.018)$, and the OR of the MTHFR A1298C genotype for $\mathrm{AC}+\mathrm{CC}$ between patients and controls was 2.100 (95\% CI, $1.149-3.837 ; P=0.015$ ). This indicates that $\mathrm{A} 1298 \mathrm{C}$ is a risk factor for childhood ALL in the Chinese population, which is in accordance with the results of previous studies $[6,7,24,26]$.

These data indicate that the MTHFR A1298C but not the C677T polymorphism is a potential biomarker for childhood ALL risk in the Chinese population, which is inconsistent with the results of Yan et al. [25] and Tong et al. [27]. We speculated that the inconsistent results in the relationship between MTHFR gene polymorphisms and childhood ALL in different studies might result from the following reasons: first, MTHFR gene distribution varied among different populations [6]; second, MTHFR polymorphisms may be affected by the level of intracellular folate, which is related to eating habits and nutritional intake; third, single SNPs provide insufficient information for comprehensive analysis of the MTHFR polymorphism; besides, other genes may regulate the susceptibility to childhood ALL, such as RFC1 80G > A and NNMT IVS $-151 \mathrm{C}>\mathrm{T}$ variants [12]. Eventually, linkage of haplotypes in the two loci would help to identify genetic markers associated with drug response differences.
The level of MTHFR $1298 \mathrm{CC}$ homozygous mutation is relatively low. In the present study, there were only two cases with CC genotypes among 98 patients. The previous studies found that the $1298 \mathrm{CC}$ homozygous mutation rate was only about $10 \%[3,12,18,20]$. Thus, we speculated that the lower number of CC cases in this study was caused by the low total number of cases in this study (98 patients and 93 controls), which may induce a high occasionality. Therefore, we combined the genotypes of AC and CC to calculate the distribution of MTHFR A1298C to improve the reliability of our findings.

It is important to note that the small sample size is a major limitation in our study because only 98 cases and 93 controls were included. However, our study is still significant for providing valid data on MTHFR C677T and A1298C genotypes for further study of MTHFR polymorphisms and childhood ALL in the Chinese population. In addition, more case-control studies with larger sample sizes and more genotypes of different ethnic pediatric populations are necessary.

\section{Conclusion}

In conclusion, we investigated the relationship between the polymorphism of MTHFR C677T and A1298C mutation and the susceptibility to childhood ALL by PCR-RFLP

Table 4 Frequencies of MTHFR A1298C polymorphisms in childhood ALL patients and controls

\begin{tabular}{|c|c|c|c|c|c|}
\hline MTHFR A1298C & Patient no. (\%) & Control no. (\%) & $x^{2 *}$ & $P$ & OR $(95 \% \mathrm{Cl})$ \\
\hline \multicolumn{6}{|l|}{ Genotype } \\
\hline AA & $54(55.1)$ & $67(72.0)$ & & - & $1.00^{\#}$ \\
\hline$A C$ & $42(42.9)$ & $25(26.9)$ & 5.628 & 0.018 & $2.084(1.131-3.841)$ \\
\hline $\mathrm{CC}$ & $2(2.0)$ & $1(1.1)$ & 0.574 & 0.449 & $2.481(0.219-28.104)$ \\
\hline$A C+C C$ & $44(44.9)$ & $26(28.0)$ & 5.898 & 0.015 & $2.100(1.149-3.837)$ \\
\hline \multicolumn{6}{|l|}{ Allele } \\
\hline A & $150(76.5)$ & $159(85.5)$ & & - & $1.00^{\#}$ \\
\hline C & $46(23.5)$ & $27(14.5)$ & 4.949 & 0.026 & $1.806(1.068-3.053)$ \\
\hline
\end{tabular}

${ }^{*}$ Corrected chi-square test; ${ }^{*}$ as reference group; OR, crude odds ratio; - , not applicable; $\mathrm{Cl}$, confidence interval. $\mathrm{AA}$, $\mathrm{AC}$ and $\mathrm{CC}$ overall distribution test, $X_{\text {test }}^{2}=5.917, P=0.05$. 
in 98 children with ALL and 93 non-ALL controls. The results indicated that the MTHFR A1298C polymorphism, not C677T, is associated with susceptibility to childhood ALL in the Chinese population. However, in the future, it will be necessary to expand the sample size and consider additional factors for exploring the relationship between the MTHFR polymorphism and the incidence of ALL to provide more accurate information for clinical research.

\section{Competing interests}

The authors declare that they have no competing interests.

\section{Authors' contributions}

$\mathrm{XL}$ and $\mathrm{QL}$ participated in the design of this study. $\mathrm{XL}$ performed the statistical analysis. XL carried out the study, together with $\mathrm{QL}$, collected important background information and drafted the manuscript. SZ collected blood samples and guided the experimental process. MC conceived this study, participated in the design and helped to draft the manuscript. All authors read and approved the final manuscript.

\section{Author details}

1Department of Pharmacy, Shanghai Children's Medical Center, Shanghai Jiaotong University School of Medicine, 1678 Dongfang Road, Pudong, Shanghai 200127, China. 'Department of Pharmacy, Nanjing Children's Hospital, Nanjing Medical University, Nanjing 210008, China.

Received: 10 August 2013 Accepted: 13 January 2014

Published: 29 January 2014

\section{References}

1. Pui C-H, Robison LL, Look AT: Acute lymphoblastic leukaemia. The Lancet 2008, 371:1030-1043.

2. Lightfoot TJ, Johnston WT, Painter D, Simpson J, Roman E, Skibola CF, Smith MT, Allan JM, Taylor GM: Genetic variation in the folate metabolic pathway and risk of childhood leukemia. Blood 2010, 115:3923-3929.

3. Atashrazm F, Zaker F, Aghaeipour M, Pazhakh V: Polymorphisms of the methylene tetrahydrofolate reductase and susceptibility to acute lymphoblastic leukemia in children. Lab Med 2011, 42:275-279.

4. Conter V, Bartram CR, Valsecchi MG, Schrauder A, Panzer-Grümayer R, Möricke A, Aricò M, Zimmermann M, Mann G, De Rossi G: Molecular response to treatment redefines all prognostic factors in children and adolescents with B-cell precursor acute lymphoblastic leukemia: results in 3184 patients of the AIEOP-BFM ALL 2000 study. Blood 2010, 115:3206-3214.

5. Nicoloso MS, Sun H, Spizzo R, Kim H, Wickramasinghe P, Shimizu M, Wojcik SE, Ferdin J, Kunej T, Xiao L: Single-nucleotide polymorphisms inside microRNA target sites influence tumor susceptibility. Cancer Res 2010, 70:2789-2798.

6. Zanrosso CW, Hatagima A, Emerenciano M, Ramos F, Figueiredo A, Félix TM, Segal SL, Giugliani R, Muniz MTC, Pombo-de-Oliveira MS: The role of methylenetetrahydrofolate reductase in acute lymphoblastic leukemia in a Brazilian mixed population. Leuk Res 2006, 30:477-481.

7. Alcasabas P, Ravindranath Y, Goyette G, Haller A, del Rosario L, Lesaca-Medina MY, Darga L, Ostrea EM, Taub JW, Everson RB: 5, 10methylenetetrahydrofolate reductase (MTHFR) polymorphisms and the risk of acute lymphoblastic leukemia (ALL) in Filipino children. Pediatr Blood Cancer 2008, 51:178-182.

8. Castro R, Rivera I, Ravasco P, Camilo M, Jakobs C, Blom H, De Almeida I: 5, 10-methylenetetrahydrofolate reductase (MTHFR) $677 \mathrm{C} \rightarrow \mathrm{T}$ and $1298 \mathrm{~A} \rightarrow \mathrm{C}$ mutations are associated with DNA hypomethylation. J Med Genet 2004, 41:454-458.

9. Ogino S, Wilson RB: Genotype and haplotype distributions of MTHFR 677C > T and 1298A > C single nucleotide polymorphisms: a meta-analysis. J Hum Genet 2003, 48:0001-0007.

10. Heijmans BT, Boer JM, Suchiman HE, Cornelisse CJ, Westendorp RG, Kromhout D, Feskens EJ, Slagboom PE: A common variant of the methylenetetrahydrofolate reductase gene (1p36) is associated with an increased risk of cancer. Cancer Res 2003, 63:1249-1253.

11. Chatzidakis K, Goulas A, Athanassiadou-Piperopoulou F, Fidani L, Koliouskas D, Mirtsou V: Methylenetetrahydrofolate reductase C677T polymorphism: Association with risk for childhood acute lymphoblastic leukemia and response during the initial phase of chemotherapy in greek patients. Pediatr Blood Cancer 2006, 47:147-151.

12. de Jonge R, Tissing WJ, Hooijberg JH, Jansen G, Kaspers GJ, Lindemans J, Peters GJ, Pieters R: Polymorphisms in folate-related genes and risk of pediatric acute lymphoblastic leukemia. Blood 2009, 113:2284-2289.

13. Oliveira E, Alves S, Quental S, Ferreira F, Norton L, Costa V, Amorim A, Prata MJ: The MTHFR C677T and A1298C polymorphisms and susceptibility to childhood acute lymphoblastic leukemia in Portugal. J Pediatr Hematol Oncol 2005, 27:425-429.

14. Krajinovic M, Lamothe $S$, Labuda D, Lemieux-Blanchard $E$, Theoret $Y$, Moghrabi A, Sinnett D: Role of MTHFR genetic polymorphisms in the susceptibility to childhood acute lymphoblastic leukemia. Blood 2004, 103:252-257.

15. Reddy H, Jamil K: Polymorphisms in the MTHFR gene and their possible association with susceptibility to childhood acute lymphocytic leukemia in an Indian population. Leuk Lymphoma 2006, 47:1333-1339.

16. Chiusolo P, Reddiconto G, Cimino G, Sica S, Fiorini A, Farina G, Vitale A, Sorà F, Laurenti L, Bartolozzi F: Methylenetetrahydrofolate reductase genotypes do not play a role in acute lymphoblastic leukemia pathogenesis in the Italian population. Haematol 2004, 89:139-144.

17. Schnakenberg E, Mehles A, Cario G, Rehe K, Seidemann K, Schlegelberger B, Elsner HA, Welte KH, Schrappe M, Stanulla M: Polymorphisms of methylenetetrahydrofolate reductase (MTHFR) and susceptibility to pediatric acute lymphoblastic leukemia in a German study population. BMC Med Genet 2005, 6:23.

18. Nikbakht M, MalekZadeh K, Kumar Jha A, Askari M, Marwaha R, Kaul D, Kaur J: Polymorphisms of MTHFR and MTR genes are not related to susceptibility to childhood ALL in North India. Exp Oncol 2012, 34:43-48.

19. Azhar MR, Rahimi Z, Vaisi-Raygani A, Akramipour R, Madani H, Parsian A: Lack of association between MTHFR C677T and A1298C polymorphisms and risk of childhood acute lymphoblastic leukemia in the Kurdish population from Western Iran. Genet Test Mol Biomarkers 2012, 16:198-202.

20. Skibola CF, Smith MT, Kane E, Roman E, Rollinson S, Cartwright RA, Morgan G: Polymorphisms in the methylenetetrahydrofolate reductase gene are associated with susceptibility to acute leukemia in adults. Proc Natl Acad Sci 1999, 96:12810-12815.

21. Franco RF, Simões BP, Tone LG, Gabellini SM, Zago MA, Falcão RP: The methylenetetrahydrofolate reductase C677T gene polymorphism decreases the risk of childhood acute lymphocytic leukaemia. $\mathrm{Br} J$ Haematol 2001, 115:616-618.

22. Aplenc R, Thompson J, Han P, La M, Zhao H, Lange B, Rebbeck T: Methylenetetrahydrofolate reductase polymorphisms and therapy response in pediatric acute lymphoblastic leukemia. Cancer Res 2005, 65:2482-2487

23. Pereira TV, Rudnicki M, Pereira AC, Pombo-de-Oliveira MS, Franco RF: 5, 10-Methylenetetrahydrofolate reductase polymorphisms and acute lymphoblastic leukemia risk: a meta-analysis. Cancer Epidemiol Biomarkers Prev 2006, 15:1956-1963.

24. Kim NK, Chong SY, Jang MJ, Hong SH, Kim HS, Cho EK, Lee JA, Ahn MJ, Kim CS, Oh D: Association of the methylenetetrahydrofolate reductase polymorphism in Korean patients with childhood acute lymphoblastic leukemia. Anticancer Res 2006, 26:2879-2881.

25. Yan J, Yin M, Dreyer ZE, Scheurer ME, Kamdar K, Wei Q, Okcu MF: A meta-analysis of MTHFR C677T and A1298C polymorphisms and risk of acute lymphoblastic leukemia in children. Pediatr Blood Cancer 2012, 58:513-518

26. Kim Yl: Methylenetetrahydrofolate reductase polymorphisms, folate, and cancer risk: a paradigm of gene-nutrient interactions in carcinogenesis. Nutr Rev 2000, 58:205-209.

27. Tong N, Fang Y, Li J, Wang M, Lu Q, Wang S, Tian Y, Rong L, Sun J, Xu J: Methylenetetrahydrofolate reductase polymorphisms, serum methylenetetrahydrofolate reductase levels, and risk of childhood acute lymphoblastic leukemia in a Chinese population. Cancer Sci 2010, 101:782-786.

doi:10.1186/2047-783X-19-5

Cite this article as: Li et al:: Association of methylenetetrahytrofolate reductase (MTHFR) C677T and A1298C polymorphisms with the susceptibility of childhood acute lymphoblastic leukaemia (ALL) in Chinese population. European Journal of Medical Research 2014 19:5. 